\title{
ON DEEP FROBENIUS DESCENT AND FLAT BUNDLES
}

\author{
Holger Brenner and Almar Kaid
}

\begin{abstract}
Let $R$ be an integral domain of finite type over $\mathbb{Z}$ and let $f: \mathcal{X} \rightarrow \operatorname{Spec} R$ be a smooth projective morphism of relative dimension $d \geq 1$. We investigate, for a vector bundle $\mathcal{E}$ on the total space $\mathcal{X}$, under what arithmetical properties of a sequence $\left(\mathfrak{p}_{n}, e_{n}\right)_{n \in \mathbb{N}}$, consisting of closed points $\mathfrak{p}_{n}$ in Spec $R$ and Frobenius descent data $\mathcal{E}_{\mathfrak{p}_{n}} \cong$ $F^{e_{n} *}(\mathcal{F})$ on the closed fibers $\mathcal{X}_{\mathfrak{p}_{n}}$, the bundle $\mathcal{E}_{0}$ on the generic fiber $\mathcal{X}_{0}$ is semistable.
\end{abstract}

Mathematical Subject Classification (2000): 14H60.

Keywords: semistable vector bundle, flat vector bundle, Frobenius morphism, Frobenius descent, finite field, Hilbert-Kunz multiplicity, relative curve.

\section{Introduction}

This paper is a continuation of our note [4]. Let $\mathbb{Z} \subseteq R$ be an integral domain of finite type and let $f: \mathcal{X} \rightarrow \operatorname{Spec} R$ be a smooth projective morphism of relative dimension $d \geq 1$. For a locally free sheaf $\mathcal{E}$ on $\mathcal{X}$ we are interested how properties of $\mathcal{E}_{0}=\left.\mathcal{E}\right|_{\mathcal{X}_{0}}$ on $\mathcal{X}_{0}=\mathcal{X} \times_{\operatorname{Spec} R} \operatorname{Spec} Q(R)$ (in characteristic zero) are related to properties of $\mathcal{E}_{\mathfrak{p}}$ on $\mathcal{X}_{\mathfrak{p}}=\mathcal{X}_{\kappa(\mathfrak{p})}=\mathcal{X} \times{ }_{\text {Spec } R}$ Spec $\kappa(\mathfrak{p})$ for closed points $\mathfrak{p}$ with finite residue class field (in positive characteristic). More specifically, we study what Frobenius descent properties for $\mathcal{E}_{\mathfrak{p}}$ force $\mathcal{E}_{0}$ to be semistable. We say that $\mathcal{E}_{\mathfrak{p}}$ admits an eth Frobenius descent if $\mathcal{E}_{\mathfrak{p}} \cong F^{e^{*}}(\mathcal{F})$ for some locally free sheaf $\mathcal{F}$ on $\mathcal{X}_{\mathfrak{p}}, F$ being the absolute Frobenius morphism. In [4] we gave, addressing a question of K. Joshi [10], an example of a bundle on a (generically) smooth projective relative curve over Spec $\mathbb{Z}$ such that $\mathcal{E}_{p}$ admits a first Frobenius descent $(e=1)$ for infinitely many prime numbers $p$, yet $\mathcal{E}_{0}$ is not semistable.

Here we allow the Frobenius exponents $e$ to grow. We will consider Frobenius descent sequences $\left(\mathfrak{p}_{n}, e_{n}\right)_{n \in \mathbb{N}}$ consisting of closed points $\mathfrak{p}_{n}$ and natural numbers $e_{n}$ such that $\mathcal{E}_{\mathfrak{p}_{n}}$ admits an $e_{n}$ th Frobenius descent. It turns out for $\mathcal{X}$ being a relative curve that one has to compare the growth of $e_{n}$ with the number of elements in the residue class fields $\kappa\left(\mathfrak{p}_{n}\right)$. Our main positive result, Theorem 3.3, states that $\mathcal{E}_{0}$ is semistable if $e_{n}-\left|\kappa\left(\mathfrak{p}_{n}\right)\right|^{c} \rightarrow \infty$, where $c$ is a constant depending on the genus of the curve and the rank of the bundle. We will give examples of Frobenius descent sequences where the characteristic is constant and $e_{n} \rightarrow \infty$, but $\mathcal{E}_{0}$ is not semistable (Examples 4.3 and 4.5).

We give a quick overview for the organization of this paper: In Section 2 we deal with bundles over varieties over a field of fixed positive characteristic. We recall the notions of strong semistability, flat bundles (introduced by Gieseker), Frobenius descent and Frobenius periodicity. We exhibit how these concepts are related over a

Received by the editors December 18, 2007. 
finite field and how they differ in general. In Section 3 we prove after some preparatory work our main theorem, and Section 4 presents the examples.

\section{Flat bundles, Frobenius descent and strong semistability}

To begin with we recall some essential notions regarding semistable sheaves on a smooth projective variety $X$ over an algebraically closed field $K$ (for a complete account see the book [9]). A coherent torsion-free sheaf $\mathcal{E}$ on $X$ is semistable (in the sense of Mumford and Takemoto) if for every coherent subsheaf $0 \neq \mathcal{F} \subset \mathcal{E}$ the inequality $\mu(\mathcal{F})=\operatorname{deg}(\mathcal{F}) / \operatorname{rk}(\mathcal{F}) \leq \operatorname{deg}(\mathcal{E}) / \operatorname{rk}(\mathcal{E})=\mu(\mathcal{E})$ holds. The sheaf $\mathcal{E}$ is stable if the inequality is always strict. The degree of a sheaf $\mathcal{F}$ is defined using intersection theory and a fixed ample invertible sheaf $\mathcal{O}_{X}(1)$ as $\operatorname{deg}(\mathcal{F})=\operatorname{deg}\left(c_{1}(\mathcal{F}) \cdot \mathcal{O}_{X}(1)^{\operatorname{dim}(X)-1}\right)$. For every coherent torsion-free sheaf $\mathcal{E}$ there exists the Harder-Narasimhan filtration $\mathcal{E}_{1} \subset \mathcal{E}_{2} \subset \ldots \subset \mathcal{E}_{t}=\mathcal{E}$ such that $\mathcal{E}_{i} / \mathcal{E}_{i-1}$ is semistable and $\mu\left(\mathcal{E}_{1}\right)>\mu\left(\mathcal{E}_{2} / \mathcal{E}_{1}\right)>$ $\ldots>\mu\left(\mathcal{E} / \mathcal{E}_{t-1}\right)$. The slopes $\mu\left(\mathcal{E}_{1}\right)$ and $\mu\left(\mathcal{E} / \mathcal{E}_{t-1}\right)$ are also denoted by $\mu_{\max }(\mathcal{E})$ and $\mu_{\min }(\mathcal{E})$ respectively. If $K$ is not algebraically closed, then we define the terms degree, semistable, etc. via the algebraic closure of $K$.

On a smooth projective variety $X$ over a field $K$ of positive characteristic $p>0$ we consider the absolute Frobenius morphism $F: X \rightarrow X$ which is the identity on the topological space $X$ and the $p$ th power map $(-)^{p}$ on the structure sheaf $\mathcal{O}_{X}$. A coherent torsion-free sheaf $\mathcal{E}$ is called strongly semistable if all Frobenius pullbacks $F^{e^{*}}(\mathcal{E})$ are semistable. The Frobenius pull-back of an algebraic cycle $Z_{i} \in$ $A^{i}(X)$ of codimension $i$ is $p^{i} Z_{i}$. Hence the degree of $F^{*}(\mathcal{E})$ with respect to $\mathcal{O}_{X}(1)$ is $\operatorname{deg}\left(c_{1}\left(F^{*}(\mathcal{E})\right) \cdot \mathcal{O}_{X}(1)^{\operatorname{dim}(X)-1}\right)=p \operatorname{deg}(\mathcal{F})$.

Definition 2.1. Let $X$ be a smooth projective variety over a field of positive characteristic and let $\mathcal{E}$ be a locally free sheaf on $X$. We say that $\mathcal{E}$ admits an eth Frobenius descent if there exists a locally free sheaf $\mathcal{F}$ such that $\mathcal{E} \cong F^{e *}(\mathcal{F})$. We say that $\mathcal{E}$ admits an infinite Frobenius descent if $\mathcal{E} \cong F^{e^{*}}\left(\mathcal{F}_{e}\right)$ holds for every $e \in \mathbb{N}$ and locally free sheaves $\mathcal{F}_{e}$. Furthermore, the locally free sheaf $\mathcal{E}$ is called flat, if there exists a collection $\left(\mathcal{F}_{e}\right)_{e \in \mathbb{N}}$ of locally free sheaves such that $\mathcal{F}_{0}=\mathcal{E}$ and $\mathcal{F}_{e} \cong F^{*}\left(\mathcal{F}_{e+1}\right)$.

The notion of flat (sometimes called stratified) bundles was introduced by Gieseker (cf. [7, Definition 1.1]). We emphasize that the notion of flatness depends on the base field (see Example 2.8). If in positive characteristic a semistable vector bundle $\mathcal{E}$ admits a Frobenius descent $\mathcal{E} \cong F^{*}(\mathcal{F})$ then clearly $\mathcal{F}$ has to be semistable too. The following easy observation is well-known (cf. [6, Lemma 5]), but for completeness we present it with a proof.

Lemma 2.2. Let $X$ be a smooth projective variety defined over a field of positive characteristic $p>0$, let $\mathcal{O}_{X}(1)$ be a fixed ample line bundle and let $\mathcal{E}$ be a locally free sheaf of degree zero and rank $r$ on $X$ with $b:=\mu_{\max }(\mathcal{E})$. If $\mathcal{E} \cong F^{e^{*}}(\mathcal{F})$ holds for some $e>\log _{2}(r b)$ and a locally free sheaf $\mathcal{F}$ then $\mathcal{F}$ is semistable. Moreover, if $\mathcal{E}$ is a flat vector bundle on $X$ then there exists an $e_{0}$ such that all descent bundles $\mathcal{F}_{e}$ are semistable for $e \geq e_{0}$.

Proof. Assume $\mathcal{F}$ is not semistable. Since by assumption $\mathcal{E}$ has degree zero and rank $r$, so has $\mathcal{F}$. Therefore, we have $\mu_{\max }(\mathcal{F}) \geq 1 / r$ and we obtain (we may assume that 
$\operatorname{dim}(X) \geq 1)$

$$
\mu_{\max }\left(F^{e *}(\mathcal{F})\right) \geq \frac{p^{e}}{r} \geq \frac{2^{e}}{r}>\frac{2^{\log _{2}(r b)}}{r}=b .
$$

This yields a contradiction. For a flat vector bundle $\mathcal{E}$ we have by definition $\mathcal{F}_{e} \cong$ $F^{*}\left(\mathcal{F}_{e+1}\right)$ for every $e \in \mathbb{N}$. Hence, the supplement follows now from the equalities $\mathcal{E}=\mathcal{F}_{0}=F^{*}\left(\mathcal{F}_{1}\right)=F^{*}\left(F^{*}\left(\mathcal{F}_{2}\right)\right)=F^{2^{*}}\left(\mathcal{F}_{2}\right)=\ldots=F^{e^{*}}\left(\mathcal{F}_{e}\right)$ for every $e>0$.

Proposition 2.3. Let $X$ be a smooth projective variety defined over a finite field $K$ and let $\mathcal{E}$ be a locally free sheaf of rank $r$ on $X$. Then the following conditions are equivalent:

(1) $\mathcal{E} \cong F^{e *}(\mathcal{E})$ for some $e>0$.

(2) There exists an étale cover $\psi: \tilde{X} \rightarrow X$ such that $\psi^{*}(\mathcal{E}) \cong \mathcal{O}_{\tilde{X}}^{r}$.

$(3) \mathcal{E}$ is a flat vector bundle.

(4) $\mathcal{E}$ admits infinite Frobenius descent.

Proof. (1) $\Leftrightarrow(2)$. This follows from [13, Satz 1.4]; see also Remark 2.7.

$(1) \Rightarrow(3)$. We define $\mathcal{F}_{i}:=F^{(-i \bmod e)^{*}}(\mathcal{E})($ taking $0 \leq-i \bmod e<e)$ and get

$$
F^{*}\left(\mathcal{F}_{i+1}\right)=F^{*}\left(F^{((-i-1) \bmod e)^{*}}(\mathcal{E})\right)=\left\{\begin{array}{l}
F^{e^{*}}(\mathcal{E}) \cong \mathcal{E} \cong \mathcal{F}_{i} \text { if } i \equiv 0 \bmod e, \\
F^{(-i \bmod e)^{*}}(\mathcal{E}) \cong \mathcal{F}_{i} \text { if } i \not \equiv 0 \bmod e
\end{array}\right.
$$

and see that $\mathcal{E}$ has the structure of a flat vector bundle.

$(3) \Rightarrow(4)$. As mentioned in the proof of Lemma 2.2 we have $\mathcal{E}=F^{e^{*}}\left(\mathcal{F}_{e}\right)$ for every $e>0$ which yields infinite Frobenius descent.

$(4) \Rightarrow(1)$. By assumption there exist locally free sheaves $\mathcal{F}_{e}, e \in \mathbb{N}$, such that $\mathcal{E} \cong F^{e^{*}}\left(\mathcal{F}_{e}\right)$ for every $e>0$. This implies the equalities $c_{i}(\mathcal{E})=p^{e i} c_{i}\left(\mathcal{F}_{e}\right)$ for the Chern classes of $\mathcal{E}$ for all $e, p=\operatorname{char}(K), i=1, \ldots, \operatorname{dim} X$. Hence, all the Chern classes $c_{i}(\mathcal{E})$ and $c_{i}\left(\mathcal{F}_{e}\right)$ are numerically trivial for $i=1, \ldots, \operatorname{dim} X$ and $e \geq 0$, because $\operatorname{deg}\left(c_{i}(\mathcal{E}) \cdot Z\right)=\operatorname{deg}\left(p^{e i} c_{i}\left(\mathcal{F}_{e}\right) \cdot Z\right)=p^{e i} \operatorname{deg}\left(c_{i}\left(\mathcal{F}_{e}\right) . Z\right)$ for every algebraic cycle $Z$ of dimension $i$. By Lemma 2.2 the bundles $\mathcal{F}_{e}$ are semistable for $e$ sufficiently large. Since the family of semistable vector bundles of fixed rank with numerically trivial Chern classes is bounded by Langer's theorem [15, Theorem 4.2] and since we are working over a finite field, there are only finitely many isomorphism classes of such bundles. Hence we must have a repetition $\mathcal{F}_{t} \cong \mathcal{F}_{s}$ for $t>s$. Applying $F^{t^{*}}$ yields

$$
\mathcal{E} \cong F^{t^{*}}\left(\mathcal{F}_{t}\right) \cong F^{t^{*}}\left(\mathcal{F}_{s}\right) \cong F^{(t-s)^{*}}\left(F^{s *}\left(\mathcal{F}_{s}\right)\right) \cong F^{(t-s)^{*}}(\mathcal{E})
$$

and we obtain a repetition of the vector bundle $\mathcal{E}$.

In [7, Theorem 1.10] D. Gieseker proved that if every flat bundle on a smooth projective variety $X$ over an algebraically closed field $K$ is trivial then the algebraic fundamental group $\pi_{1}(X)$ is trivial. It is an open question in [7, Beginning of $\left.\S 2\right]$ whether the converse is true (and was proven for projective spaces and $K 3$ surfaces in $[7$, Theorem 2.2 and Theorem 2.3]). For bundles which are flat over a finite field we have the following converse.

Corollary 2.4. Let $X$ be a smooth projective variety defined over a finite field $K$ with $\pi_{1}\left(X_{\bar{K}}\right)=0$. Then every flat bundle on $X$ is trivial. 
Proof. Let $\mathcal{E}$ be a flat bundle on $X$. By Proposition 2.3 there exists an étale cover $\psi: \tilde{X} \rightarrow X$ such that $\psi^{*}(\mathcal{E})$ becomes trivial on $\tilde{X}$. Hence $\psi^{*}(\mathcal{E})_{\bar{K}}$ is trivial on $\tilde{X}_{\bar{K}}$. By assumption $\pi_{1}\left(X_{\bar{K}}\right)$ is trivial and therefore $X_{\bar{K}}$ is simply connected (cf. [17, Theorem 5.3]). Hence $\mathcal{E}_{\bar{K}}$ is trivial on $X_{\bar{K}}$ and so $\mathcal{E}$ is trivial on $X$. [13].

We also present a weaker version of Proposition 2.3 which goes essentially back to

Proposition 2.5. Let $X$ be a smooth projective variety defined over a finite field $K$ and let $\mathcal{E}$ be a locally free sheaf. Then the following conditions are equivalent.

(1) There exists a repetition $F^{t^{*}}(\mathcal{E}) \cong F^{s *}(\mathcal{E})$ with $t>s$.

$(2) \mathcal{E}$ is strongly semistable (with respect to every $\mathcal{O}_{X}(1)$ ) with numerically trivial Chern classes.

(3) There exists an étale cover $\psi: \tilde{X} \rightarrow X$ such that $\psi^{*}\left(F^{s^{*}}(\mathcal{E})\right)$ becomes trivial for a certain $s>0$.

Proof. $(1) \Rightarrow(2)$. The repetition yields the equations $p^{t i} c_{i}(\mathcal{E})=p^{s i} c_{i}(\mathcal{E})$ for $i=$ $1, \ldots, \operatorname{dim}(X)$, hence $p^{(t-s) i} c_{i}(\mathcal{E})=c_{i}(\mathcal{E})$ and the assertion on the Chern classes follows. In particular, $\operatorname{deg}(\mathcal{E})=0$ for every $\mathcal{O}_{X}(1)$. We have $F^{t *}(\mathcal{E})=F^{(t-s) *}\left(F^{s *}(\mathcal{E})\right) \cong$ $F^{s^{*}}(\mathcal{E})$ and so $F^{k(t-s) *}\left(F^{s *}(\mathcal{E})\right) \cong F^{s^{*}}(\mathcal{E})$ for all $k$. Thus $F^{{ }^{*}}(\mathcal{E})$ is semistable by Lemma 2.2. Every $F^{e^{*}}(\mathcal{E})$ has a Frobenius pull-back which is isomorphic to $F^{{ }^{*}}(\mathcal{E})$, hence $\mathcal{E}$ is strongly semistable.

$(2) \Rightarrow(1)$. This follows also immediately from the boundedness of the family of semistable vector bundles with numerically trivial Chern classes.

$(1) \Leftrightarrow(3)$. For this equivalence see [13, Satz 1.4].

Corollary 2.6. Let $X$ be a smooth projective variety defined over a finite field $K$ and let $\mathcal{E}$ be a flat vector bundle on $X$. Then $\mathcal{E}$ is strongly semistable.

Proof. This follows from Proposition 2.3 and Proposition 2.5.

Remark 2.7. The implications $(1) \Rightarrow(2) \Rightarrow(3) \Rightarrow(4)$ of Proposition 2.3 and $(1) \Rightarrow(3) \Rightarrow(2)$ of Proposition 2.5 also hold for smooth projective varieties defined over an arbitrary field of positive characteristic. The cited result [13, Satz 1.4] of Lange and Stuhler holds over a finite field, but not in the generality stated there. An example of Y. Laszlo mentioned in [2, after Theorem 1.1] shows that for a semistable étale trivializable vector bundle $\mathcal{E}$ we do not necessarily have a Frobenius periodicity $F^{e^{*}}(\mathcal{E}) \cong \mathcal{E}$ for an $e>0$. We provide also such an example in 2.10. It was also shown in [2, Theorem 1.1] that the theorem of Lange and Stuhler is true if $\mathcal{E}$ is stable.

A flat vector bundle $\mathcal{E}$ over an infinite field of positive characteristic is in general not semistable. Examples for this behavior were constructed in [6] by D. Gieseker.

In the following we give examples of flat and strongly semistable bundles without any Frobenius repetitions.

Example 2.8. Let $K$ be an algebraically closed field of positive characteristic $p$ and let $X$ be a smooth projective variety defined over $K$. If $\mathcal{L} \in \operatorname{Pic}^{0}(X)$ is a line bundle algebraically equivalent to zero then $\mathcal{L}$ is flat $\operatorname{since} \operatorname{Pic}^{0}(X)$ is $p$-divisible; see $[22$, Application II.6.2]. Suppose furthermore that $K$ contains a transcendental element and $\mathcal{L}$ is non-torsion in $\operatorname{Pic}^{0}(X)$. Then all powers $F^{e^{*}}(\mathcal{L}) \cong \mathcal{L}^{p^{e}}$ are distinct for 
$e \geq 0$. Hence, we obtain a flat vector bundle of rank one without any repetitions. By $[2$, Theorem 1.1] $\mathcal{L}$ is not étale trivializable.

In contrast, if $X$ is defined over a finite field $K$ such that the $p$-torsion of $\operatorname{Pic}^{0}(X)$ is non-trivial, we find a line bundle $\mathcal{L} \neq \mathcal{O}_{X}$ such that $\mathcal{L}^{p} \cong \mathcal{O}_{X}$. Hence, there is no repetition $\mathcal{L} \cong \mathcal{L}^{p^{e}}$ for any $e>0$. By Proposition 2.3 the line bundle $\mathcal{L}$ can not have infinite Frobenius descent. In particular, the property of being flat depends on the base field.

The following proposition gives a method to construct flat vector bundles of rank two without any Frobenius repetition. Unlike the previous example these bundles are étale trivializable and hence come by [13, Proposition 1.2] from a representation $\rho: \pi_{1}(X) \rightarrow G L_{2}(K)$ of the algebraic fundamental group $\pi_{1}(X)$.

Proposition 2.9. Let $X$ be a smooth projective variety defined over an algebraically closed field $K$ of characteristic $p>0$ which contains transcendental elements. If the $p$-rank of $X$ is at least two then there exist non-trivial extensions of $\mathcal{O}_{X}$ by $\mathcal{O}_{X}$ which are flat, strongly semistable and without any Frobenius repetitions.

Proof. The Frobenius morphism acts on $V:=H^{1}\left(X, \mathcal{O}_{X}\right) p$-linearly, i.e., $F^{*}(\lambda c)=$ $\lambda^{p} F^{*}(c)$ for all $\lambda \in K$ and $c \in V$. We decompose $V=V_{s} \oplus V_{n}$ in its semi-simple part $V_{s}$, where there exists a basis $v_{1}, \ldots, v_{k}$ such that $F^{*}\left(v_{i}\right)=v_{i}$ for all $i=1, \ldots, k$, and its nilpotent part $V_{n}$, where $F^{*}$ is a nilpotent map (cf. [22, III.14, last corollary]). The assumption on the $p$-rank of $X$ means that $\operatorname{dim}_{K} V_{s} \geq 2$. We fix two linearly independent vectors $v$ and $w$ in $V_{s}$ with $F^{*}(v)=v$ and $F^{*}(w)=w$, and we consider the cohomology class $c=v+t w$, where $t \in K^{\times}$is a transcendental element.

Let the vector bundle $\mathcal{E}$ be the extension of $\mathcal{O}_{X}$ by $\mathcal{O}_{X}$ given by $c \in H^{1}\left(X, \mathcal{O}_{X}\right)=$ $\operatorname{Ext}^{1}\left(\mathcal{O}_{X}, \mathcal{O}_{X}\right)$. Such a bundle is strongly semistable. The eth Frobenius pull-back of $\mathcal{E}$ is the extension given by $F^{e^{*}}(c)=v+t^{p^{e}} w$. The same reasoning shows that $\mathcal{E}$ itself is the $n$th Frobenius pull-back of the extension given by $v+t^{1 / p^{n}} w$ (where $t^{1 / p^{n}}$ exists since $K$ is algebraically closed). Hence $\mathcal{E}$ is flat. Assume $F^{{ }^{*}}(\mathcal{E}) \cong F^{e^{*}}(\mathcal{E})$ with $s>e$. Then the corresponding cohomology classes must differ by a scalar, say $v+t^{p^{s}} w=\lambda\left(v+t^{p^{e}} w\right)$. Hence $\lambda=1$ and so $t^{p^{s}}=t^{p^{e}}$ and $t^{p^{s-e}}=1$, contradicting the transcendence of $t$.

Example 2.10. We consider the Fermat quartic

$$
C:=\operatorname{Proj}\left(\overline{\mathbb{F}_{5}(t)}[X, Y, Z] /\left(X^{4}+Y^{4}-Z^{4}\right)\right)
$$

over the algebraic closure of the function field $\mathbb{F}_{5}(t)$. The Cech-cohomology classes $\frac{\sqrt[4]{2} Z^{3}}{X^{2} Y}$ and $\frac{\sqrt[4]{2} Z^{3}}{X Y^{2}}$ are linearly independent in $V:=H^{1}\left(C, \mathcal{O}_{C}\right)$. The Frobenius acts on $\frac{\sqrt[4]{2} Z^{3}}{X^{2} Y}$ as follows:

$$
\begin{aligned}
F^{*}\left(\frac{\sqrt[4]{2} Z^{3}}{X^{2} Y}\right) & =\frac{2 \sqrt[4]{2} Z^{15}}{X^{10} Y^{5}}=\frac{2 \sqrt[4]{2} Z^{3} Z^{12}}{X^{10} Y^{5}} \\
& =\frac{2 \sqrt[4]{2} Z^{3}\left(X^{12}+3 X^{8} Y^{4}+3 X^{3} Y^{8}+Y^{12}\right)}{X^{10} Y^{5}} \\
& =\frac{\sqrt[4]{2} Z^{3}\left(X^{8} Y^{4}\right)}{X^{10} Y^{5}}=\frac{\sqrt[4]{2} Z^{3}}{X^{2} Y}
\end{aligned}
$$


and analogously we obtain $F^{*}\left(\frac{\sqrt[4]{2} Z^{3}}{X Y^{2}}\right)=\frac{\sqrt[4]{2} Z^{3}}{X Y^{2}}$ by symmetry, so $\frac{\sqrt[4]{2} Z^{3}}{X^{2} Y}$ and $\frac{\sqrt[4]{2} Z^{3}}{X Y^{2}}$ are classes which are fixed under the Frobenius. Hence the bundle defined by $c:=$ $\frac{\sqrt[4]{2} Z^{3}}{X^{2} Y}+t \frac{\sqrt[4]{2} Z^{3}}{X Y^{2}}$ has all properties described in Proposition 2.9. The descent data are given by the extensions defined by $c_{n}:=\frac{\sqrt[4]{2} Z^{3}}{X^{2} Y}+\sqrt[5]{t} \frac{\sqrt[4]{2} Z^{3}}{X Y^{2}}, n \geq 0$.

The following example was used in [5] to show that tight closure does not commute with localization. It also shows that strong semistability is not an open property for geometric deformations (see also [14, Example 2.11] for another instructive example), though the existence of a Frobenius repetition is. We will come back to this example in Lemma 4.2 and Example 4.3. For a homogeneous coordinate ring $R$ of a projective variety $X$ and homogeneous elements $f_{1}, \ldots, f_{n} \in R$ of degrees $d_{i}:=\operatorname{deg}\left(f_{i}\right), i=$ $1, \ldots, n$, such that the ideal generated by the $f_{i}$ is $R_{+}$-primary, the syzygy bundle $\operatorname{Syz}\left(f_{1}, \ldots, f_{n}\right)(m)$ is given by the short exact sequence

$$
0 \longrightarrow \operatorname{Syz}\left(f_{1}, \ldots, f_{n}\right)(m) \longrightarrow \bigoplus_{i=1}^{n} \mathcal{O}_{X}\left(m-d_{i}\right) \stackrel{f_{1}, \ldots, f_{n}}{\longrightarrow} \mathcal{O}_{X}(m) \longrightarrow 0 .
$$

It has rank $n-1$ and degree $\left((n-1) m-\sum_{i=1}^{n} d_{i}\right) \operatorname{deg}\left(\mathcal{O}_{X}(1)\right)$.

Example 2.11. We consider the smooth plane curve

$$
C:=\operatorname{Proj}\left(\mathbb{F}_{2}(T)[X, Y, Z] /\left(Z^{4}+X Y Z^{2}+X^{3} Z+Y^{3} Z+\left(T+T^{2}\right) X^{2} Y^{2}\right)\right),
$$

where $T$ might be algebraic (but $T \neq 0,1$ ) or transcendental. We consider the syzygy bundle $\operatorname{Syz}\left(X^{2}, Y^{2}, Z^{2}\right)(3)$ and its Frobenius pull-backs

$$
\operatorname{Syz}\left(X^{2 q}, Y^{2 q}, Z^{2 q}\right)(3 q) \cong F^{e *}\left(\operatorname{Syz}\left(X^{2}, Y^{2}, Z^{2}\right)(3)\right),
$$

$q=2^{e}$. For $T$ transcendental all these pull-backs are semistable, but this is false for every algebraic value; see [20, Theorem 4.13] and [3, Corollary 4.6]. In the transcendental case there are however no repetitions in the sequence $F^{e *}\left(\operatorname{Syz}\left(X^{2}, Y^{2}, Z^{2}\right)(3)\right)$. If there would be such a repetition then this would also hold for almost all algebraic values, contradicting the fact that in the algebraic case the syzygy bundle $\operatorname{Syz}\left(X^{2}, Y^{2}, Z^{2}\right)(3)$ is not strongly semistable. We do not know whether the bundle $\operatorname{Syz}\left(X^{2}, Y^{2}, Z^{2}\right)(3)$ is flat for $T$ transcendental.

Example 2.12. Let $C$ be a smooth projective curve of genus two defined over an algebraically closed field $K$ of characteristic 3. It was shown in [16, Corollary 6.6] that the rational map

$$
V: \mathcal{M}_{C}\left(2, \mathcal{O}_{C}\right) \rightarrow \mathcal{M}_{C}\left(2, \mathcal{O}_{C}\right),[\mathcal{E}] \longmapsto\left[F^{*}(\mathcal{E})\right]
$$

(also called the Verschiebung) from the moduli space $\mathcal{M}_{C}\left(2, \mathcal{O}_{C}\right)$ parameterizing semistable rank two vector bundles with trivial determinant is surjective. This means for a stable rank two vector bundle $\mathcal{E}$ that $\mathcal{E} \cong F^{*}(\mathcal{F})$, where $\mathcal{F}$ is necessarily stable too. Hence every stable rank two vector bundle is flat.

For a semistable but not stable bundle $\mathcal{E}$ we obtain just sequences $[\mathcal{E}]=\left[\mathcal{F}_{0}\right]=$ $\left[F^{*}\left(\mathcal{F}_{1}\right)\right]=\left[F^{2 *}\left(\mathcal{F}_{2}\right)\right]=\ldots=\left[F^{n *}\left(\mathcal{F}_{n}\right)\right]$ of $S$-equivalence classes for every $n \geq 0$ such that $\left[\mathcal{F}_{n}\right]=\left[F^{*}\left(\mathcal{F}_{n+1}\right)\right]$. Such an $S$-flat bundle is in general not flat. For instance, let $C$ be an elliptic curve and consider the bundle $F_{2}$ in Atiyah's classification [1], 
i.e., the unique indecomposable sheaf of rank two and degree zero with $\Gamma\left(C, F_{2}\right) \cong K$. The bundle $F_{2}$ is sitting inside the short exact sequence

$$
0 \longrightarrow \mathcal{O}_{C} \longrightarrow F_{2} \longrightarrow \mathcal{O}_{C} \longrightarrow 0
$$

and we see that $\left[F_{2}\right]=\left[\mathcal{O}_{C}^{2}\right]$. In particular, $F_{2}$ is $S$-flat. If the Hasse invariant of $C$ is zero, we have $F^{*}\left(F_{2}\right) \cong \mathcal{O}_{C}^{2}$. Therefore, $F_{2}$ is not flat since it is not a pull-back of another vector bundle. If the Hasse invariant of $C$ equals one, then $F^{*}\left(F_{2}\right) \cong F_{2}$ and $F_{2}$ is flat.

\section{Deep Frobenius descent on relative curves}

In this section we consider a smooth projective morphism $f: \mathcal{X} \rightarrow \operatorname{Spec} R$ of schemes of relative dimension $d \geq 1$ where the $\operatorname{ring} R$ is Noetherian. In this situation we denote by $\mathcal{X}_{\mathfrak{p}}:=\mathcal{X} \times_{\operatorname{Spec} R} \operatorname{Spec} \kappa(\mathfrak{p})$ the fiber over the prime ideal $\mathfrak{p} \in \operatorname{Spec} R$, where $\kappa(\mathfrak{p}):=R_{\mathfrak{p}} / \mathfrak{p} R_{\mathfrak{p}}$ denotes the residue class field at the point $\mathfrak{p}$. These fibers are smooth projective schemes of dimension $d$. Further, we fix an $f$-very ample line bundle $\mathcal{O}_{\mathcal{X}}(1)$ on $\mathcal{X}$, in particular $\left.\mathcal{O}_{\mathcal{X}}(1)\right|_{\mathcal{X}_{\mathfrak{p}}}$ is a very ample line bundle on $\mathcal{X}_{\mathfrak{p}}$. If $\mathcal{E}$ is a locally free sheaf on $\mathcal{X}$ then $\mathcal{E}_{\mathfrak{p}}:=\left.\mathcal{E}\right|_{\mathcal{X}_{\mathrm{p}}}$ denotes the restriction of $\mathcal{E}$ to the fiber $\mathcal{X}_{\mathfrak{p}}$.

The following Lemma is well-known, but since it is essential for our further progress we will present it with a full proof.

Lemma 3.1. Let $R$ be a Noetherian ring and let $f: \mathcal{X} \rightarrow \operatorname{Spec} R$ be a smooth projective morphism of relative dimension $d \geq 1$ together with a fixed $f$-very ample line bundle $\mathcal{O}_{\mathcal{X}}(1)$. If $\mathcal{E}$ is a locally free sheaf on $\mathcal{X}$ then there exists a global bound $b$ such that $\mu_{\max }\left(\mathcal{E}_{\mathfrak{p}}\right) \leq b$ for all $\mathfrak{p} \in \operatorname{Spec} R$.

Proof. Without loss of generality, we can assume that $\operatorname{Spec} R$ is connected. The locally free sheaf $\mathcal{E}(m)$ is globally generated for $m \gg 0$ (cf. [8, Theorem 5.17]), i.e., we have a surjective morphism

$$
\mathcal{O}_{\mathcal{X}}^{s}(-m) \longrightarrow \mathcal{E} \longrightarrow 0
$$

for some $s>0$. Restriction to each fiber $\mathcal{X}_{\mathfrak{p}}, \mathfrak{p} \in \operatorname{Spec} R$, yields

$$
\mathcal{O}_{\mathcal{X}_{\mathfrak{p}}}^{s}(-m) \longrightarrow \mathcal{E}_{\mathfrak{p}} \longrightarrow 0 \text {. }
$$

Hence $\mu_{\min }\left(\mathcal{E}_{\mathfrak{p}}\right) \geq-m \operatorname{deg}\left(\mathcal{O}_{\mathcal{X}}(1)\right)$. In particular, this bound holds for the slope of every quotient sheaf of $\mathcal{E}_{\mathfrak{p}}$. Therefore, if $\mathcal{E}_{\mathfrak{p}}$ is not semistable and $\mathcal{F}$ is the maximal destabilizing subsheaf of $\mathcal{E}_{\mathfrak{p}}$ we obtain the simple inequalities

$$
\begin{aligned}
\mu_{\max }\left(\mathcal{E}_{\mathfrak{p}}\right) & =\mu(\mathcal{F})=\frac{\operatorname{deg}(\mathcal{F})}{\operatorname{rk}(\mathcal{F})}=\frac{\operatorname{deg}\left(\mathcal{E}_{\mathfrak{p}}\right)}{\operatorname{rk}(\mathcal{F})}-\frac{\operatorname{deg}\left(\mathcal{E}_{\mathfrak{p}} / \mathcal{F}\right)}{\operatorname{rk}(\mathcal{F})} \\
& =\frac{\operatorname{deg}(\mathcal{E})}{\operatorname{rk}(\mathcal{F})}-\frac{\operatorname{rk}\left(\mathcal{E}_{\mathfrak{p}} / \mathcal{F}\right)}{\operatorname{rk}(\mathcal{F})} \mu\left(\mathcal{E}_{\mathfrak{p}} / \mathcal{F}\right) \leq \frac{\operatorname{deg}(\mathcal{E})}{\operatorname{rk}(\mathcal{F})}+\frac{\operatorname{rk}\left(\mathcal{E}_{\mathfrak{p}} / \mathcal{F}\right)}{\operatorname{rk}(\mathcal{F})} m \operatorname{deg}\left(\mathcal{O}_{\mathcal{X}}(1)\right) \\
& \leq \max (0, \operatorname{deg}(\mathcal{E}))+\operatorname{rk}(\mathcal{E}) m \operatorname{deg}\left(\mathcal{O}_{\mathcal{X}}(1)\right)
\end{aligned}
$$

where $\operatorname{deg}(\mathcal{E})=\operatorname{deg}\left(\mathcal{E}_{\mathfrak{p}}\right)$ is constant in the family (cf. [9, Definition 1.2.11] and [22, Section II.5]). This bound also holds for $\mathcal{E}_{\mathfrak{p}}$ semistable. 
Lemma 3.2. Let $C$ be a smooth projective curve defined over a finite field $K$. Then the number of isomorphism classes of semistable vector bundles of rank $r$ and degree zero is bounded from above by $|K|^{c}$, where $c$ is a constant depending only on $r$ and the genus of $C$.

Proof. Let $\mathcal{E}$ be a semistable vector bundle on $C$ of rank $r$ and degree zero. Further, we fix an ample line bundle $\mathcal{O}(1)$ of degree one. For $\ell>2 g-1$ we have $\mu(\mathcal{E}(\ell))=\ell>$ $2 g-1$. Therefore, by [24, Lemme 20], the bundle $\mathcal{E}(\ell)$ is generated by global sections and $h^{1}(C, \mathcal{E}(\ell))=0$. So we obtain a short exact sequence

$$
0 \longrightarrow \mathcal{F} \longrightarrow \mathcal{O}(-\ell)^{s} \longrightarrow \mathcal{E} \longrightarrow 0
$$

where $s:=h^{0}(C, \mathcal{E}(\ell))=r \ell+r(1-g)$ and $\mathcal{F}$ is a locally free sheaf of rank $s-r$ and degree $-\ell s$. Let $0=\mathcal{F}_{0} \subset \mathcal{F}_{1} \subset \ldots \subset \mathcal{F}_{t}=\mathcal{F}$ be the Harder-Narasimhan filtration of $\mathcal{F}$. Since $\mu\left(\mathcal{F}_{i}\right) \leq-\ell$ holds for all $i=1, \ldots, t$, the degrees of the subbundles $\mathcal{F}_{i}$ are all negative. Thus we have $\operatorname{deg}\left(\mathcal{F} / \mathcal{F}_{t-1}\right) \geq \operatorname{deg}(\mathcal{F})=-\ell s$ and moreover $\mu\left(\mathcal{F} / \mathcal{F}_{t-1}\right) \geq-\ell s$. This yields

$$
\begin{aligned}
\mu\left(\left(\mathcal{F} / \mathcal{F}_{t-1}\right)(\ell(s+1))\right) & =\mu\left(\mathcal{F} / \mathcal{F}_{t-1}\right)+\ell(s+1) \\
& \geq-\ell s+\ell(s+1) \\
& =\ell .
\end{aligned}
$$

So for $k:=\ell(s+1)$, again by [24, Lemme 20], all twisted quotients $\left(\mathcal{F}_{i} / \mathcal{F}_{i-1}\right)(k)$, $i=1, \ldots, t$, are globally generated with $h^{1}\left(C,\left(\mathcal{F}_{i} / \mathcal{F}_{i-1}\right)(k)\right)=0$.

We show now by induction that the bundles $\mathcal{F}_{i}(k)$, and in particular $\mathcal{F}(k)$, are also globally generated with $h^{1}\left(C, \mathcal{F}_{i}(k)\right)=0$. To do that, we look at the short exact sequences

$$
0 \longrightarrow \mathcal{F}_{i-1}(k) \longrightarrow \mathcal{F}_{i}(k) \longrightarrow\left(\mathcal{F}_{i} / \mathcal{F}_{i-1}\right)(k) \longrightarrow 0 .
$$

By the induction hypothesis $\mathcal{F}_{i-1}(k)$ is generated by its global sections and we have $h^{1}\left(C, \mathcal{F}_{i-1}(k)\right)=0$ which implies $h^{1}\left(C, \mathcal{F}_{i}(k)\right)=0$ too, since the quotients do not have higher cohomology. Hence, we obtain a commutative diagram

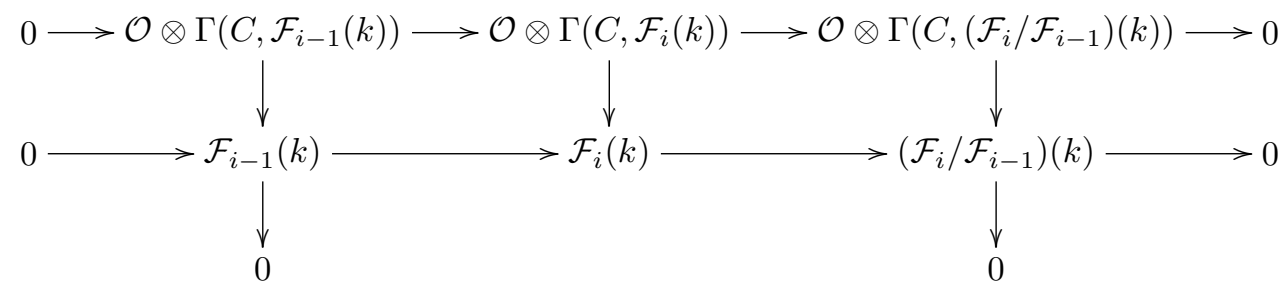

with exact rows and columns. We have to show that the morphism of sheaves in the middle is surjective too. But, since surjectivity is a local property, we can consider this diagram locally at a point $P \in C$. Then the surjectivity follows from the five lemma (cf. [23, $\S 42$, Aufgabe 14]). So we have proved that the bundle $\mathcal{F}(k)$ is globally 
generated and we have the following commutative diagram

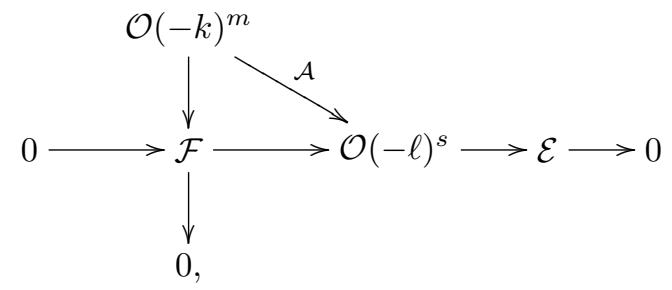

where $m:=h^{0}(C, \mathcal{F}(k))=-\ell s+(s-r) k+(s-r)(1-g)$ and $\mathcal{A}$ is an $m \times s$ matrix with entries in $\Gamma(C, \mathcal{O}(k-\ell))=\Gamma(C, \mathcal{O}(\ell s))$. So every semistable vector bundle of rank $r$ and degree zero is parameterized by such a matrix $\mathcal{A}$. The $K$-vector space $\Gamma(C, \mathcal{O}(\ell s))$ has by Riemann-Roch dimension $h^{0}(C, \mathcal{O}(\ell))=\ell s+1-g=: n$. Hence $|K|^{c}$, with $c:=n m s$, is an upper bound for the number of possible matrices and we see that the constant $c$ only depends on $r$ and $g$.

We are now able to prove our main theorem. We assume from now on that $R$ is a $\mathbb{Z}$-domain of finite type, $\mathbb{Z} \subseteq R$, and that $f: C \rightarrow \operatorname{Spec} R$ is a smooth projective relative curve. In this situation the residue class field $\kappa(\mathfrak{p})$ of every closed point in Spec $R$ is a finite field. We denote by $C_{0}$ the fiber of the relative curve over the generic point $(0) \in \operatorname{Spec} R$ (in characteristic zero).

For a vector bundle $\mathcal{E}$ on $C$ we ask when arithmetical Frobenius descent data imply the semistability of $\mathcal{E}_{0}$ on $C_{0}$. Such data are given by a sequence $\left(\mathfrak{p}_{n}, e_{n}\right)_{n \in \mathbb{N}}$, where $\mathfrak{p}_{n}$ are closed points in Spec $R$ and $e_{n}$ describes the depth of the Frobenius descent on $C_{\mathfrak{p}_{n}}$, i.e., $\mathcal{E}_{\mathfrak{p}_{n}} \cong F^{e_{n} *}\left(\mathcal{F}_{n}\right)$, with $\mathcal{F}_{n}$ locally free on $C_{\mathfrak{p}_{n}}$. We call such a sequence a Frobenius descent sequence.

Theorem 3.3. Let $f: C \rightarrow \operatorname{Spec} R$ be a smooth projective relative curve together with a fixed $f$-very ample line bundle $\mathcal{O}_{C}(1)$, where $R$ is a $\mathbb{Z}$-domain of finite type, $\mathbb{Z} \subseteq R$. Further let $\mathcal{E}$ be a locally free sheaf of rank $r$ on $C$. Suppose there exists a Frobenius descent sequence $\left(\mathfrak{p}_{n}, e_{n}\right)_{n \in \mathbb{N}}$ and assume $\left(e_{n}-\left|\kappa\left(\mathfrak{p}_{n}\right)\right|^{c}\right)_{n \in \mathbb{N}} \rightarrow \infty$, where c is the constant from Lemma 3.2. Then $\mathcal{E}_{0}$ is semistable on the generic fiber $C_{0}$.

Proof. Let $\mathcal{F}_{n}$ be locally free on $C_{\mathfrak{p}_{n}}$ such that $\mathcal{E}_{\mathfrak{p}_{n}} \cong F^{e_{n}} *\left(\mathcal{F}_{n}\right)$. First of all we remark that the degree of $\mathcal{E}$ has to be zero. This follows, $\operatorname{since} \operatorname{deg}\left(\mathcal{E}_{\mathfrak{p}_{n}}\right)=p_{n}^{e_{n}} \operatorname{deg}\left(\mathcal{F}_{n}\right)$ holds for $p_{n}=\operatorname{char}\left(\kappa\left(\mathfrak{p}_{n}\right)\right)$ and $e_{n} \rightarrow \infty$ (compare this also with the proof of $[4$, Theorem 3.1]). By Lemma 3.2 the number of isomorphism classes of semistable vector bundles of degree zero and rank $r$ on each fiber $C_{\mathfrak{p}_{n}}$ is bounded from above by $\left|\kappa\left(\mathfrak{p}_{n}\right)\right|^{c}$, where the constant $c$ depends only on $r$ and the genus of $C$ and is therefore independent of the closed point $\mathfrak{p}_{n}$. Since by assumption the sequence $\left(e_{n}-\left|\kappa\left(\mathfrak{p}_{n}\right)\right|^{c}\right)_{n \in \mathbb{N}}$ diverges, we fix an index $n$ such that

$$
e_{n}-\left|\kappa\left(\mathfrak{p}_{n}\right)\right|^{c} \geq t:=\log _{2}(r b),
$$

where $b$ is the bound of Lemma 3.1. We apply Lemma 2.2 to $\mathcal{E}_{\mathfrak{p}_{n}} \cong F^{e_{n}} *\left(\mathcal{F}_{n}\right)=$ $F^{\left(e_{n}-k\right)^{*}}\left(F^{k^{*}}\left(\mathcal{F}_{n}\right)\right)$ to conclude that $F^{k^{*}}\left(\mathcal{F}_{n}\right)$ is semistable for all $k$ with $e_{n}-k>t$. Due to the inequality $e_{n}-t \geq\left|\kappa\left(\mathfrak{p}_{n}\right)\right|^{c}$ there has to be a repetition $F^{k^{*}}\left(\mathcal{F}_{n}\right) \cong$ $F^{k^{\prime *}}\left(\mathcal{F}_{n}\right), k<k^{\prime}<e_{n}-t$. By Proposition 2.5 the locally free sheaf $\mathcal{F}_{n}$ is strongly 
semistable on $C_{\mathfrak{p}_{n}}$. Hence $\mathcal{E}_{\mathfrak{p}_{n}}$ is strongly semistable too. By the openness of semistability we obtain the semistability of $\mathcal{E}_{0}=\left.\mathcal{E}\right|_{C_{0}}$ on the generic fiber $C_{0}$.

Corollary 3.4. In the situation of Theorem 3.3 assume that there exists a closed point $\mathfrak{p} \in \operatorname{Spec} R$ such that $\mathcal{E}_{\mathfrak{p}}$ admits an infinite Frobenius descent on $C_{\mathfrak{p}}$, i.e., we have $e_{n} \rightarrow \infty$ in the sequence $\left(\mathfrak{p}, e_{n}\right)_{n \in \mathbb{N}}$. Then $\mathcal{E}_{0}$ is semistable on the generic fiber $C_{0}$.

Proof. Since $\mathfrak{p}_{n}=\mathfrak{p}$ for all $n$, the expression $\left|\kappa\left(\mathfrak{p}_{n}\right)\right|^{c}$ is constant, so this follows from Theorem 3.3. This corollary can also be proved using Proposition 2.3, by which the locally free sheaf $\mathcal{E}_{\mathfrak{p}}$ is strongly semistable on $C_{\mathfrak{p}}$. Again by the openness of semistability we conclude that $\mathcal{E}_{0}$ is semistable on the generic fiber $C_{0}$.

Remark 3.5. In our proof of Theorem 3.3 we have found a closed point $\mathfrak{p}=\mathfrak{p}_{n}$ in Spec $R$ where $\mathcal{E}_{\mathfrak{p}}$ is strongly semistable. Moreover, $\mathcal{E}_{\mathfrak{p}}$ is even flat. We have $\mathcal{E}_{\mathfrak{p}} \cong$ $F^{e_{n} *}\left(\mathcal{F}_{n}\right)$ and $F^{k^{*}}\left(\mathcal{F}_{n}\right) \cong F^{k^{\prime *}}\left(\mathcal{F}_{n}\right), k<k^{\prime}<e_{n}-t$. Hence

$$
\mathcal{E}_{\mathfrak{p}} \cong F^{\left(e_{n}-k\right)^{*}}\left(F^{k^{*}}\left(\mathcal{F}_{n}\right)\right) \cong F^{\left(e_{n}-k\right)^{*}}\left(F^{k^{\prime *}}\left(\mathcal{F}_{n}\right)\right) \cong F^{\left(k^{\prime}-k\right)^{*}}\left(F^{e_{n} *}\left(\mathcal{F}_{n}\right)\right) \text {, }
$$

and $\mathcal{E}_{\mathfrak{p}}$ is flat by Proposition 2.3. A problem of Miyaoka ([18, Problem 5.4]) asks whether for $\mathcal{E}_{0}$ semistable the set $S$ of primes $\mathfrak{p}$ of positive residue characteristic in Spec $R$ with strongly semistable reduction $\mathcal{E}_{\mathfrak{p}}$ is dense. It is also open whether for $\mathcal{E}_{0}$ semistable there exists always a closed point $\mathfrak{p}$ such that $\mathcal{E}_{\mathfrak{p}}$ is flat (or whether there exists a Frobenius descent sequence fulfilling the arithmetic condition of Theorem $3.3)$.

Example 3.6. Suppose that $D \rightarrow \operatorname{Spec} \mathbb{F}_{p}$ is a smooth projective curve and $\mathcal{M} \neq \mathcal{O}_{D}$ is an invertible sheaf with $\mathcal{M}^{p} \cong \mathcal{O}_{D}$. Consider $C=D \times_{\operatorname{Spec}} \mathbb{F}_{p}$ Spec $\mathbb{F}_{p}[T] \rightarrow$ Spec $\mathbb{F}_{p}[T]$ and $\mathcal{L}=\mathcal{M} \times{ }_{D} C$. For $\mathfrak{p} \in \operatorname{Spec} \mathbb{F}_{p}[T]$, the sheaf $\mathcal{L}_{\mathfrak{p}}$ is strongly semistable on $C_{\mathfrak{p}}=D_{\kappa(\mathfrak{p})}$. However, it is not flat at any closed point; this follows from Proposition 2.3. For every $n$ there exists $q=p^{a_{n}}$ and an invertible sheaf $\mathcal{N}$ on $D_{\mathbb{F}_{q}}$ with $\mathcal{L}_{\mathbb{F}_{q}} \cong \mathcal{N}^{p^{n}}$, because this is true on $D_{\bar{F}_{p}}$. Hence we have a Frobenius descent sequence $\left(\mathfrak{p}_{n}, n\right)$ with $\kappa\left(\mathfrak{p}_{n}\right)=\mathbb{F}_{p^{a_{n}}}$. We have $n-p^{c a_{n}} \leq 0$ for all $n$, because else there were a repetition $\mathcal{N}^{p^{e^{\prime}}}=\mathcal{N}^{p^{e}}$ for $e<e^{\prime} \leq n$.

Remark 3.7. The existence of a Frobenius descent for $\mathcal{E}_{\mathfrak{p}}$ is by the so-called Cartiercorrespondence [11, Theorem 5.1] equivalent to the existence of an integrable connection $\nabla_{\mathfrak{p}}$ on $\mathcal{E}_{\mathfrak{p}}$ with $p$-curvature zero (see [11] and [12] for a detailed exposition of these notions). We neither know whether these connections are related to each other nor whether they come from a global connection $\nabla$ on $\mathcal{E}$. The GrothendieckKatz $p$-curvature conjecture [12, (I quat)] states that given an integrable connection $\nabla$ on $\mathcal{E}\left(\right.$ on $\mathcal{X} \rightarrow \operatorname{Spec} R$ ) with the property that $\left.\nabla\right|_{\mathcal{E}_{\mathfrak{p}}}$ on $\mathcal{X}_{\mathfrak{p}}$ has $p$-curvature zero for $\operatorname{char}(\kappa(\mathfrak{p})) \gg 0$, then there exists an étale cover $\psi: Y \rightarrow \mathcal{X}_{0}$ such that $\left(\psi^{*}\left(\mathcal{E}_{0}\right), \psi^{*}\left(\nabla_{0}\right)\right)$ is trivial. This is only possible if $\mathcal{E}_{0}$ is semistable.

\section{Counterexamples for the case of constant characteristic}

In this section we give several examples consisting of rank two vector bundles $\mathcal{E}$ on smooth projective relative curves $C$ and Frobenius descent sequences $\left(\mathfrak{p}_{n}, e_{n}\right)_{n \in \mathbb{N}}$ with certain arithmetical properties, but where $\mathcal{E}_{0}$ is not semistable on the generic 
fiber $C_{0}$. We first recall briefly our example presented in [4] of a Frobenius descent sequence $\left(\mathfrak{p}_{n}, e_{n}\right)_{n \in \mathbb{N}}=\left(\left(p_{n}\right), 1\right)_{n \in \mathbb{N}}$ where $p_{n}$ runs through infinitely many prime numbers. Hence Theorem 3.3 does not hold when the sequence $\left(e_{n}\right)_{n \in \mathbb{N}}$ is constant and $\operatorname{char} \kappa\left(\mathfrak{p}_{n}\right) \rightarrow \infty$. In Example 4.3 and Example 4.5 we present Frobenius descent sequences of closed points of constant residue characteristic and $e_{n} \rightarrow \infty$, where again $\mathcal{E}_{0}$ is not semistable. Hence infinite Frobenius descent in different points of the same residue characteristic does not imply semistability. These examples are based on examples by P. Monsky showing how the Hilbert-Kunz multiplicity depends on algebraic parameters, and use the geometric interpretation of Hilbert-Kunz theory in terms of (strong) semistability. We do not know of an example where $\left|\kappa\left(\mathfrak{p}_{n}\right)\right| \rightarrow \infty$ and $e_{n} \geq\left|\kappa\left(\mathfrak{p}_{n}\right)\right|$ and where $\mathcal{E}_{0}$ is not semistable.

Example 4.1. Consider the smooth relative Fermat curve

$$
C:=\operatorname{Proj}\left(\mathbb{Z}_{d}[X, Y, Z] /\left(X^{d}+Y^{d}+Z^{d}\right)\right) \longrightarrow \operatorname{Spec} \mathbb{Z}_{d}
$$

of degree $d=2 \ell+1$ and $\ell \geq 2$. The first Frobenius pull-back of the syzygy bundle $\operatorname{Syz}\left(X^{2}, Y^{2}, Z^{2}\right)(3)$ is sitting inside the short exact sequence

$$
0 \longrightarrow \mathcal{O}_{C_{p}}(\ell-1) \longrightarrow \operatorname{Syz}\left(X^{2 p}, Y^{2 p}, Z^{2 p}\right)(3 p) \longrightarrow \mathcal{O}_{C_{p}}(-\ell+1) \longrightarrow 0
$$

on the fibers $C_{p}$ for primes of the form $p \equiv \ell(d)$ (see [4, Lemma 2.1]). As a consequence of the Cartier-correspondence this sequence does not split for almost all such $p$ (see [4, Lemma 2.4]). Therefore, we take the vector bundle $\mathcal{E}$ on $C$ defined by the extension corresponding to the non-trivial Cech-cohomology class $c=Z^{d-1} / X Y \in$ $H^{1}\left(C, \mathcal{O}_{C}(d-3)\right)$. On the fibers $C_{p}$ we have (because $\operatorname{dim} H^{1}\left(C_{p}, \mathcal{O}_{C_{p}}(d-3)\right)=1$ ) the equality $c=\lambda c^{\prime}$, where $c^{\prime}$ is the class corresponding to our short exact sequence above, hence $\mathcal{E}_{p} \cong F^{*}\left(\operatorname{Syz}\left(X^{2}, Y^{2}, Z^{2}\right)(3)\right)$. But the restriction $\mathcal{E}_{0}$ to the generic fiber $C_{0}$ is not semistable.

Lemma 4.2. Let $G:=Z^{4}+X Y Z^{2}+Z\left(X^{3}+Y^{3}\right)+\left(T+T^{2}\right) X^{2} Y^{2} \in \mathbb{F}_{2}[T][X, Y, Z]$ be the equation defining the relative projective curve

$$
C:=\operatorname{Proj}\left(\mathbb{F}_{2}[T][X, Y, Z] /(G)\right) \longrightarrow \operatorname{Spec} \mathbb{F}_{2}[T]=\mathbb{A}_{\mathbb{F}_{2}}^{1} .
$$

Further let $\mathfrak{p} \in \operatorname{Spec} \mathbb{F}_{2}[T]$ be a closed point corresponding to an algebraic value $T \mapsto$ $t \in \overline{\mathbb{F}}_{2} t \neq 0,1$, and define $d:=\left[\mathbb{F}_{2}(t): \mathbb{F}_{2}\right]$. Then the Harder-Narasimhan filtration of the dth Frobenius pull-back of $\operatorname{Syz}\left(X^{2}, Y^{2}, Z^{2}\right)(3)$ is given by the short exact sequence

$$
0 \longrightarrow \mathcal{O}_{C_{\mathfrak{p}}}(1) \longrightarrow \operatorname{Syz}\left(X^{2^{d+1}}, Y^{2^{d+1}}, Z^{2^{d+1}}\right)\left(3 \cdot 2^{d}\right) \longrightarrow \mathcal{O}_{C_{\mathfrak{p}}}(-1) \longrightarrow 0
$$

on the fiber $C_{\mathfrak{p}}$.

Proof. To prove the Lemma we use, as in [4, Remark 2.2], Hilbert-Kunz theory and its geometric approach (cf. [3] and [25]). P. Monsky has shown in [20, Theorem 4.13] that the Hilbert-Kunz multiplicity (see [19]) of the homogeneous coordinate ring $R_{\mathfrak{p}}$ of the fiber $C_{\mathfrak{p}}$ (which is smooth for $t \neq 0,1$ ) equals

$$
e_{H K}\left(R_{\mathfrak{p}}\right)=\left\{\begin{array}{l}
3 \text { if } \kappa(\mathfrak{p})=\mathbb{F}_{2}(T), \\
3+\frac{1}{4^{d}} \text { if } \kappa(\mathfrak{p})=\mathbb{F}_{2}(t) \subset \overline{\mathbb{F}}_{2}
\end{array}\right.
$$

By [3, Corollary 4.6(ii)] the restriction $\left.\Omega_{\mathbb{P}^{2}}\right|_{C_{\mathfrak{p}}} \cong \operatorname{Syz}(X, Y, Z)$ of the cotangent bundle on the projective plane to the fiber $C_{\mathfrak{p}}$ is strongly semistable for the generic point $\mathfrak{p} \in \operatorname{Spec} \mathbb{F}_{2}[T]$ corresponding to a transcendental value and not strongly semistable 
for all algebraic instances. Monsky further proved in [20, Theorem 3.1] that the $d+1$ th pull-back $F^{(d+1)^{*}}(\operatorname{Syz}(X, Y, Z))\left(\frac{3 q}{2}-1\right) \cong \operatorname{Syz}\left(X^{q}, Y^{q}, Z^{q}\right)\left(\frac{3 q}{2}-1\right), q=2^{d+1}$, has a non-trivial section. We have to show that this section has no zeros. Consider the Harder-Narasimhan filtration

$$
0 \longrightarrow \mathcal{L} \longrightarrow \operatorname{Syz}\left(X^{q}, Y^{q}, Z^{q}\right) \longrightarrow \tilde{\mathcal{L}} \longrightarrow 0
$$

of $\operatorname{Syz}\left(X^{q}, Y^{q}, Z^{q}\right)$ where $\mathcal{L}$ is a line bundle of degree $\operatorname{deg}(\mathcal{L})=-6 q+\alpha, \alpha>0$, and the quotient $\tilde{\mathcal{L}}$ is a line bundle of $\operatorname{degree} \operatorname{deg}(\tilde{\mathcal{L}})=-6 q-\alpha$ (we have to have $\left.\operatorname{deg}(\mathcal{L})+\operatorname{deg}(\tilde{\mathcal{L}})=-12 q=\operatorname{deg}\left(\operatorname{Syz}\left(X^{q}, Y^{q}, Z^{q}\right)\right)\right)$. By [3, Corollary 4.6] we can compute $e_{H K}\left(R_{\mathfrak{p}}\right)$ from the short exact sequence above which constitutes the strong Harder-Narasimhan filtration in the sense of [15, Paragraph 2.6] and [3, Section 1] since we are dealing with rank two vector bundles. We obtain $e_{H K}\left(R_{\mathfrak{p}}\right)=3+\frac{\alpha^{2}}{4 \cdot 4^{d+1}}=$ $3+\frac{1}{4^{d}}$ which yields $\alpha=4$. Hence, $\mathcal{L} \otimes_{\mathcal{O}_{C_{\mathfrak{p}}}} \mathcal{O}_{C_{\mathfrak{p}}}\left(\frac{3 q}{2}-1\right)$ has degree zero and a nontrivial section, so $\mathcal{L} \cong \mathcal{O}_{C_{\mathfrak{p}}}\left(1-\frac{3 q}{2}\right)$ and $\tilde{\mathcal{L}} \cong \mathcal{O}_{C_{\mathfrak{p}}}\left(-\frac{3 q}{2}-1\right)$. Eventually, we obtain that

$$
0 \longrightarrow \mathcal{O}_{C_{\mathfrak{p}}}(1) \longrightarrow \operatorname{Syz}\left(X^{q}, Y^{q}, Z^{q}\right)\left(3 \cdot 2^{d}\right) \longrightarrow \mathcal{O}_{C_{\mathfrak{p}}}(-1) \longrightarrow 0
$$

constitutes the Harder-Narasimhan filtration of $\operatorname{Syz}\left(X^{q}, Y^{q}, Z^{q}\right)\left(\frac{3 q}{2}\right)$.

Example 4.3. In the following we work over the smooth projective relative curve

$$
C:=\operatorname{Proj}\left(\mathbb{Z}[T]_{P(T)}[X, Y, Z] /(G)\right) \longrightarrow \operatorname{Spec} \mathbb{Z}[T]_{P(T)},
$$

where $G$ is defined as in Lemma 4.2 and where $P(T)$ ensures that the relative curve is smooth (2 is not a factor of $P(T)$ ). We consider the vector bundle $\mathcal{E}:=$ $\mathcal{O}_{C}(1) \oplus \mathcal{O}_{C}(-1)$ and the sequence $\left(\mathfrak{p}_{d}, e_{d}\right)_{d \in \mathbb{N}}=\left(\left(2, f_{d}(T)\right), d\right)_{d \in \mathbb{N}}$ of closed points $\mathfrak{p}_{d} \in \operatorname{Spec} \mathbb{Z}[T]$ of constant residue characteristic two, where $f_{d}(T)$ denotes an irreducible polynomial of degree $d$ in $\mathbb{F}_{2}[T]$. Each fiber $C_{\mathfrak{p}_{d}}:=C \times_{\operatorname{Spec}} \mathbb{Z}[T] \operatorname{Spec} \kappa\left(\mathfrak{p}_{d}\right)$ is a smooth projective curve defined over $\mathbb{F}_{2^{d}}=\kappa\left(\mathfrak{p}_{d}\right)$. The sequence

$$
0 \longrightarrow \mathcal{O}_{C_{\mathfrak{p}_{d}}}(1) \longrightarrow \operatorname{Syz}\left(X^{2^{d+1}}, Y^{2^{d+1}}, Z^{2^{d+1}}\right)\left(3 \cdot 2^{d}\right) \longrightarrow \mathcal{O}_{C_{\mathfrak{p}_{d}}}(-1) \longrightarrow 0
$$

from Lemma 4.2 defines a cohomology class

$$
c \in H^{1}\left(C_{\mathfrak{p}_{d}}, \mathcal{O}_{C_{\mathfrak{p}_{d}}}(2)\right) \cong \operatorname{Ext}^{1}\left(\mathcal{O}_{C_{\mathfrak{p}_{d}}}(-1), \mathcal{O}_{C_{\mathfrak{p}_{d}}}(1)\right) \text {. }
$$

But by Serre-duality we have $H^{1}\left(C_{\mathfrak{p}_{d}}, \mathcal{O}_{C_{\mathfrak{p}_{d}}}(2)\right) \cong H^{0}\left(C_{\mathfrak{p}_{d}}, \mathcal{O}_{C_{\mathfrak{p}_{d}}}(-1)\right)=0$ on each fiber $C_{\mathfrak{p}_{d}}$. Hence, the sequence above splits and the class $c$ corresponds to the trivial extension $\mathcal{E}_{\mathfrak{p}_{d}} \cong \mathcal{O}_{C_{\mathfrak{p}_{d}}}(1) \oplus \mathcal{O}_{C_{\mathfrak{p}_{d}}}(-1)$. Therefore, the vector bundle $\mathcal{E}$ admits for every closed point $\mathfrak{p}_{d}$ a $d$ th Frobenius descent $\mathcal{E}_{\mathfrak{p}_{d}} \cong F^{d^{*}}\left(\operatorname{Syz}\left(X^{2}, Y^{2}, Z^{2}\right)(3)\right)$, but $\mathcal{E}$ is obviously not semistable on the generic fiber $C_{0}$. Hence $\mathcal{E}$ has arbitrarily deep Frobenius descent for a sequence of closed points of constant characteristic, and $e_{d} \rightarrow$ $\infty$. Here $e_{d}-\left|\kappa\left(\mathfrak{p}_{d}\right)\right|^{c}=d-2^{d c} \rightarrow-\infty$, and the numerical condition in Theorem 3.3 is not fulfilled.

Lemma 4.4. Let $H:=Z^{4}-X Y(X+Y)(X+T Y) \in \mathbb{F}_{3}[T][X, Y, Z]$ and consider the projective relative curve

$$
C:=\operatorname{Proj}\left(\mathbb{F}_{3}[T][X, Y, Z] /(H)\right) \longrightarrow \operatorname{Spec} \mathbb{F}_{3}[T]=\mathbb{A}_{\mathbb{F}_{3}}^{1} .
$$


Then for every closed point $\mathfrak{p} \in \operatorname{Spec} \mathbb{F}_{3}[T]$ corresponding to an algebraic value $T \mapsto t$, $t \neq 0,1$, of degree $d=\left[\mathbb{F}_{3}(t): \mathbb{F}_{3}\right]$ the $n=(d+1)$ th Frobenius pull-back of $\operatorname{Syz}(X, Y, Z)$ on $C_{\mathfrak{p}}$ is sitting inside the short exact sequence

$$
0 \longrightarrow \mathcal{O}_{C_{\mathfrak{p}}} \longrightarrow \operatorname{Syz}\left(X^{3^{n}}, Y^{3^{n}}, Z^{3^{n}}\right)(u) \longrightarrow \mathcal{O}_{C_{\mathfrak{p}}}(-3) \longrightarrow 0
$$

where $u:=\frac{3^{n+1}-3}{2}$. This sequence splits and constitutes the Harder-Narasimhan filtration of $\operatorname{Syz}\left(X^{3^{n}}, Y^{3^{n}}, Z^{3^{n}}\right)(u)$.

Proof. It follows from [25, Proposition 5.10] that $F^{e *}(\operatorname{Syz}(X, Y, Z))$ is semistable for $e=0, \ldots, d-1$ and not semistable for $e \geq d$. As usual we have $F^{n *}(\operatorname{Syz}(X, Y, Z)) \cong$ $\operatorname{Syz}\left(X^{3^{n}}, Y^{3^{n}}, Z^{3^{n}}\right)$. Let

$$
0 \longrightarrow \mathcal{L} \longrightarrow \operatorname{Syz}\left(X^{3^{n}}, Y^{3^{n}}, Z^{3^{n}}\right) \longrightarrow \tilde{\mathcal{L}} \longrightarrow 0
$$

be the Harder-Narasimhan filtration of the $n$th pull-back, where $\mathcal{L}$ and $\tilde{\mathcal{L}}$ are line bundles of degrees $\operatorname{deg}(\mathcal{L})=k+\alpha$, where $\alpha>0$, and $\operatorname{deg}(\tilde{\mathcal{L}})=k-\alpha$ with $k=$ $-2 \cdot 3^{n+1}=\operatorname{deg}\left(\operatorname{Syz}\left(X^{3^{n}}, Y^{3^{n}}, Z^{3^{n}}\right)\right) / 2$. It was shown by P. Monsky [21, Theorem III] that the Hilbert-Kunz multiplicity of the homogeneous coordinate ring $R_{\mathfrak{p}}$ of each fiber $C_{\mathfrak{p}}$ equals $e_{H K}\left(R_{\mathfrak{p}}\right)=3+\frac{1}{9^{n}}$. Hence we obtain $\alpha=6$ via [3, Corollary 4.6]. Next we tensor the short exact sequence above with $u=\frac{3^{n+1}-3}{2}$ so that $\mathcal{L}(u)$ has degree zero and $\tilde{\mathcal{L}}(u)$ has degree -12 . We have to show that $\mathcal{L}(u) \cong \mathcal{O}_{C_{\mathfrak{p}}}$. Assume, $\mathcal{L}(u)$ is not isomorphic to $\mathcal{O}_{C_{\mathfrak{p}}}$ (and so $\tilde{\mathcal{L}}(u) \neq \mathcal{O}_{C_{\mathfrak{p}}}(-3)$ ). We recall that one can compute from the presenting sequence of $\operatorname{Syz}\left(X^{3^{n}}, Y^{3^{n}}, Z^{3^{n}}\right)(m)$ the Hilbert-Kunz function via

$$
\begin{aligned}
\operatorname{length}\left(\left(R_{\mathfrak{p}} /(X, Y, Z)^{\left[3^{n}\right]}\right)_{m}\right)= & h^{0}\left(C_{\mathfrak{p}}, \mathcal{O}_{C_{\mathfrak{p}}}(m)\right)-3 h^{0}\left(C_{\mathfrak{p}}, \mathcal{O}_{C_{\mathfrak{p}}}\left(m-3^{n}\right)\right) \\
& +h^{0}\left(C_{\mathfrak{p}}, \operatorname{Syz}\left(X^{3^{n}}, Y^{3^{n}}, Z^{3^{n}}\right)(m)\right),
\end{aligned}
$$

and summing these expressions over $m \geq 0$ (where we can restrict to a finite sum, as the alternating sum is 0 for $m \gg 0)$. In fact we only have to sum up to $u+$ 3 , since by Serre-duality we have $H^{1}\left(C_{\mathfrak{p}}, \mathcal{L}(u+4)\right) \cong H^{0}\left(C_{\mathfrak{p}},(\mathcal{L}(u))^{*}(-3)\right)^{*}=0$ and $H^{1}\left(C_{\mathfrak{p}}, \tilde{\mathcal{L}}(u+4)\right) \cong H^{0}\left(C_{\mathfrak{p}},(\tilde{\mathcal{L}}(u))^{*}(-3)\right)^{*}=0$ and therefore the cohomology $H^{1}\left(C_{\mathfrak{p}}, \operatorname{Syz}\left(X^{3^{n}}, Y^{3^{n}}, Z^{3^{n}}\right)(u+4)\right)$ vanishes. For $m=u+1, u+2, u+3$ we get by Riemann-Roch (the quotient line bundle $\tilde{\mathcal{L}}(m)$ has no non-trivial global sections in these degrees):

$$
\begin{aligned}
& h^{0}\left(C_{\mathfrak{p}}, \operatorname{Syz}\left(X^{3^{n}}, Y^{3^{n}}, Z^{3^{n}}\right)(u+1)\right)=h^{0}\left(C_{\mathfrak{p}}, \mathcal{L}(u+1)\right)=2, \\
& h^{0}\left(C_{\mathfrak{p}}, \operatorname{Syz}\left(X^{3^{n}}, Y^{3^{n}}, Z^{3^{n}}\right)(u+2)\right)=h^{0}\left(C_{\mathfrak{p}}, \mathcal{L}(u+2)\right)=6, \\
& h^{0}\left(C_{\mathfrak{p}}, \operatorname{Syz}\left(X^{3^{n}}, Y^{3^{n}}, Z^{3^{n}}\right)(u+3)\right)=h^{0}\left(C_{\mathfrak{p}}, \mathcal{L}(u+3)\right)=10 .
\end{aligned}
$$

In degrees $m \leq u$ we have $h^{0}\left(C_{\mathfrak{p}}, \operatorname{Syz}\left(X^{3^{n}}, Y^{3^{n}}, Z^{3^{n}}\right)(m)\right)=0$ (for $m=u$ by assumption). The Hilbert function $H F_{R_{\mathfrak{p}}}(m)=h^{0}\left(C_{\mathfrak{p}}, \mathcal{O}_{C_{\mathfrak{p}}}(m)\right)$ of the homogeneous coordinate ring $R_{\mathfrak{p}}$ of each fiber $C_{\mathfrak{p}}$ is given by $H F_{R_{\mathfrak{p}}}(0)=1, H F_{R_{\mathfrak{p}}}(1)=3$ and $H F_{R_{\mathfrak{p}}}(m)=4 m-2$ for $m \geq 2$. So we have accumulated all data to compute the 
Hilbert-Kunz function $\varphi$ for the exponent $n=d+1$ and get:

$$
\begin{aligned}
\varphi\left(3^{n}\right) & =\sum_{m=0}^{u+3} h^{0}\left(C_{\mathfrak{p}}, \mathcal{O}_{C_{\mathfrak{p}}}(m)\right)-3 \sum_{m=0}^{u+3} h^{0}\left(C_{\mathfrak{p}}, \mathcal{O}_{C_{\mathfrak{p}}}\left(m-3^{n}\right)\right)+18 \\
& =4+\sum_{m=2}^{u+3}(4 m-2)-3\left(4+\sum_{m=2}^{u+3-3^{n}}(4 m-2)\right)+18 \\
& =2(u+3)^{2}-2-3\left(2\left(u+3-3^{n}\right)^{2}-2\right)+10 \\
& =-4(u+3)^{2}+4(u+3) 3^{n+1}-2 \cdot 3^{2 n+1}+14 \\
& =-\left(3^{n+1}+3\right)^{2}+2\left(3^{n+1}+3\right) 3^{n+1}-2 \cdot 3^{2 n+1}+14 \\
& =3^{2 n+1}+5 .
\end{aligned}
$$

But this contradicts [21, Theorem IIb], where P. Monsky proved with different methods that $\varphi\left(3^{n}\right)=3^{2 n+1}+9$. Hence, $\mathcal{L}(u) \cong \mathcal{O}_{C_{\mathfrak{p}}}$ and in particular $\tilde{\mathcal{L}}(u) \cong \mathcal{O}_{C_{\mathfrak{p}}}(-3)$. The sequence splits since $H^{1}\left(C_{\mathfrak{p}}, \mathcal{O}_{C_{\mathfrak{p}}}(3)\right)=0$.

Example 4.5. First we look at the smooth projective relative curve

$$
C:=\operatorname{Proj}\left(\mathbb{Z}[T]_{P(T)}[X, Y, Z] /(H)\right) \longrightarrow \operatorname{Spec} \mathbb{Z}[T]_{P(T)},
$$

where $H$ is defined as in Lemma 4.4 and localization at $P(T)$ makes the curve smooth (3 is not a factor of $P(T))$. We consider a sequence $\left(\mathfrak{p}_{d}\right)_{d \in \mathbb{N}}=\left(\left(3, f_{d}(T)\right)\right)_{d \in \mathbb{N}}$ of closed points in Spec $\mathbb{Z}[T]$, where $f_{d}(T)$ denotes a polynomial of degree $d$ which is irreducible in $\mathbb{F}_{3}[T]$. Unlike in Example 4.3 no twist of a pull-back of $\operatorname{Syz}(X, Y, Z)$ on a fiber $C_{\mathfrak{p}_{d}}$ has degree zero. Therefore, we pass over to a suitable finite cover of $C$. As in [5, Remark 4.4] we look at the ring homomorphism

$$
\mathbb{Z}[T][X, Y, Z] /(H) \longrightarrow \mathbb{Z}[T][U, V, W] /\left(W^{8}-\left(U^{4}+V^{4}\right)\left(U^{4}+T V^{4}\right)\right)=: B
$$

given by $X \mapsto U^{4}, Y \mapsto V^{4}, Z \mapsto U V W^{2}$ and the corresponding map $g: D:=$ $\operatorname{Proj}(B) \rightarrow C$ of smooth projective relative curves (over $\operatorname{Spec} \mathbb{Z}[T]_{P(T)}$ ). Let $\mathcal{E}:=$ $\mathcal{O}_{D}(6) \oplus \mathcal{O}_{D}(-6)$. It is easy to see that $g^{*}\left(\mathcal{O}_{C}(1)\right) \cong \mathcal{O}_{D}(4)$ and hence the pull-back under $g$ of the short exact sequence of Lemma 4.4 yields

$$
0 \longrightarrow \mathcal{O}_{D_{\mathfrak{p}_{d}}} \longrightarrow \operatorname{Syz}\left(U^{4 \cdot 3^{n}}, V^{4 \cdot 3^{n}}, U^{3^{n}} V^{3^{n}} W^{2 \cdot 3^{n}}\right)(4 u) \longrightarrow \mathcal{O}_{D_{\mathfrak{p}_{d}}}(-12) \longrightarrow 0
$$

$(n=d+1)$ on each fiber $D_{\mathfrak{p}_{d}}$. After tensoring with $\mathcal{O}_{D_{\mathfrak{p}_{d}}}(6)$ we obtain

$$
0 \longrightarrow \mathcal{O}_{D_{\mathfrak{p}_{d}}}(6) \longrightarrow F^{(d+1)^{*}}\left(\operatorname{Syz}\left(U^{4}, V^{4}, U V W^{2}\right)(6)\right) \longrightarrow \mathcal{O}_{D_{\mathfrak{p}_{d}}}(-6) \longrightarrow 0 .
$$

Because this sequence splits we get $\mathcal{E}_{\mathfrak{p}_{d}} \cong F^{(d+1)^{*}}\left(\operatorname{Syz}\left(U^{4}, V^{4}, U V W^{2}\right)(6)\right)$. Therefore $\left(\mathfrak{p}_{d}, d+1\right)_{d \in \mathbb{N}}$ is a Frobenius descent sequence.

\section{Acknowledgements}

We thank the referee and Hélène Esnault for many useful comments and Helena Fischbacher-Weitz for careful reading of this manuscript. 


\section{References}

[1] M. F. Atiyah, Vector bundles over an elliptic curve, Proc. London Math. Soc. 7 (1957) 414-452.

[2] I. Biswas and L. Ducrohet, An analog of a theorem of Lange and Stuhler for principal bundles, C.R. Acad. Sci. Paris, Ser. I 345 (2007), no. 9, 495-497.

[3] H. Brenner, The rationality of the Hilbert-Kunz multiplicity in graded dimension two, Math. Ann. 334 (2006), no. 1, 91-110.

[4] H. Brenner and A. Kaid, A remark on Frobenius descent for vector bundles, Michigan Math. J. 57 (2008) 63-69.

[5] H. Brenner and P. Monsky, Tight closure does not commute with localization, Preprint (2007). ArXiv:0710.2913.

[6] D. Gieseker, Stable vector bundles and the Frobenius morphism, Ann. Sci. Ecole Norm. Sup. (4) 6 (1973) 95-101.

[7] - Flat vector bundles and the fundamental group in non-zero characteristic, Ann. Scuola Norm. Sup. Pisa Cl. Sci. (4) 2 (1975), no. 1, 1-31.

[8] R. Hartshorne, Algebraic Geometry, Springer, New York (1977).

[9] D. Huybrechts and M. Lehn, The Geometry of Moduli Spaces of Sheaves, Viehweg (1997).

[10] K. Joshi, Some remarks on vector bundles, Preprint (2007). http://math.arizona.edu/kirti/ homepage.

[11] N. M. Katz, Nilpotent connections and the monodromy theorem: applications of a result of Turrittin, Inst. Hautes Études Sci. Publ. Math. 39 (1970) 175-232.

[12] - Algebraic solutions of differential equations (p-curvature and the Hodge filtration), Invent. Math. 18 (1972) 1-118.

[13] H. Lange and U. Stuhler, Vektorbündel auf Kurven und Darstellungen der algebraischen Fundamentalgruppe, Math. Zeitschrift 156 (1977) 73-83.

[14] A. Langer, Moduli spaces of sheaves and principal G-bundles, to appear in proceedings of AMS Summer Institute at Seattle in 2005.

[15] - Semistable sheaves in positive characteristic, Ann. Math. 159 (2004) 251-276.

[16] Y. Laszlo and C. Pauly, The Frobenius map, rank 2 vector bundles and Kummer's quartic surface in characteristic 2 and 3, Adv. Math. 185 (2005), no. 2, 246-269.

[17] J. S. Milne, Étale Cohomology, Princeton University Press (1980).

[18] Y. Miyaoka, The Chern class and Kodaira dimension of a minimal variety, in Algebraic Geometry, Sendai 1985, Vol. 10 of Adv. Stud. Pure Math., 449-476 (1987).

[19] P. Monsky, The Hilbert-Kunz function, Math. Ann. 263 (1983) 43-49.

$[20]-$, Hilbert-Kunz functions in a family: point-S $S_{4}$ quartics, J. Algebra 208 (1998), no. 1, 343-358.

[21] —, On the Hilbert-Kunz function of $z^{D}-p_{4}(x, y)$, J. Algebra 291 (2005), no. 2, 350-372.

[22] D. Mumford, Abelian Varieties, Oxford University Press (1970).

[23] G. Scheja and U. Storch, Lehrbuch der Algebra, Teil 1, Teubner, 2. Auflage edition (1994)

[24] C. S. Seshadri, Fibrés vectoriels sur les courbes algébrique, Asterisque 96 (1982)

[25] V. Trivedi, Semistability and Hilbert-Kunz multiplicity for curves, J. Algebra 284 (2005), no. 2, 627-644.

Universität Osnabrück, Fachbereich 6: Mathematik/Informatik, Albrechtstr. 28A, 49069 OSNABRÜCK

E-mail address: hbrenner@uni-osnabrueck.de

Department of Pure Mathematics, University of Sheffield, Hicks Building, Hounsfield Road, Sheffield S3 7RH, United Kingdom

E-mail address: a.kaid@sheffield.ac.uk 